\title{
Exploration on Building the Campus Information Center for Cloud Computing
}

\author{
Zhou Zi-li $^{1 *}$ and Lu Hui-juan ${ }^{2}$ \\ ${ }^{1}$ Zhejiang Institute of Mechanical \& Engineering \\ ${ }^{2}$ College of Information Engineering \\ China JiLiang University \\ ${ }^{1}$ Zhouzili88@163.com, ${ }^{2}$ hjlu@cjlu.edu.cn
}

\begin{abstract}
Cloud computing is considered as a novel pattern of information management and computing services, taken as another revolution in the information industry. This paper introduces the overall planning, function structure, security, and resource integration of the cloud-computing campus information center (C3IC) with practice. And an information resource integration model and a load baseline algorithm based on radial basis function are also proposed and discussed, to produce some rewarding experiences for C3IC's construction with very strong practicability. With the C3IC, the units in digital campus can be provided with computing-resources rent service in virtualization. Finally, based on the analysis of the latest research work on the C3IC, the significance is discussed and outlined.
\end{abstract}

Keywords: cloud computing; information center; resource integration; security

\section{Introduction}

Cloud computing (CC) is a novel pattern of information management and computing services, CC can combine distributed resources, storage and computing capacity together for management and service, which is quite different from the traditional IDC's operation and management concept. CC is being designed for dynamic resource allocation and on-demand service, with the strong capability of processing mega-data in low cost.

C3IC provides the massive information resources, with strong expandability of only several operations on a cloud platform. Once we have C3IC, all the units in university do not need to buy servers or storage any more, to avoid repeat constructions [1].

The unified information resources center can maximize the resource utilization and get the reasonable resource and the lower whole construction costs. The management of data and equipment can promote the sharing of resources in whole university, in order to efficient and convenient information resource services [2]. All the network security equipment and links are redundant, to assure safety and stabile accessing. According to

\footnotetext{
* This work was supported by the computer science major teaching supervision committee project fund of technical and vocational college under Ministry of Education (JZW590112KC35), the National Natural Science Foundation of China (No. 61272315, No.60842009), Zhejiang Provincial Natural Science Foundation (No.Y1110342) and Zhejiang provincial science and Technology Department of international cooperation project (No.2012C24030).
}

Corresponding author: ZHOU Zi-li, E-mail addresses: zhouzili88@163.com. 
the scale of cloud computing, the deployment is set up from single-point to redundant. The general plan of C3IC is shown in Figure 1.

In C3IC, all the computing resources join into the integrated computing resources pool. And the storage resources join into the integrated storage pool. All the units in university can share them. And provide storage to businesses [3]. C3IC is the carrier of digital campus data businesses, including the educational administration system, student system, One-Card-Through System, library system and so on. By the way, all the original systems are migrated to the C3IC [4].

C3IC's server resources are integrated through virtualization, to meet the need of the rapid deployment. And the storage resources can also be integrated through virtualization to support units' need. With internal politics, computing and storage resources are dynamically allocated according to the requests of business systems [5]. When the resources are not enough, it can be dynamically allocated to meet the new need.

The paper is structured as follows: Section II describes the related Function modules. Section III introduces the Analysis of the system safety. Section IV presents the information resource integration demonstration. Section V gives the experiment. Finally, we summarize the work in Section VI.

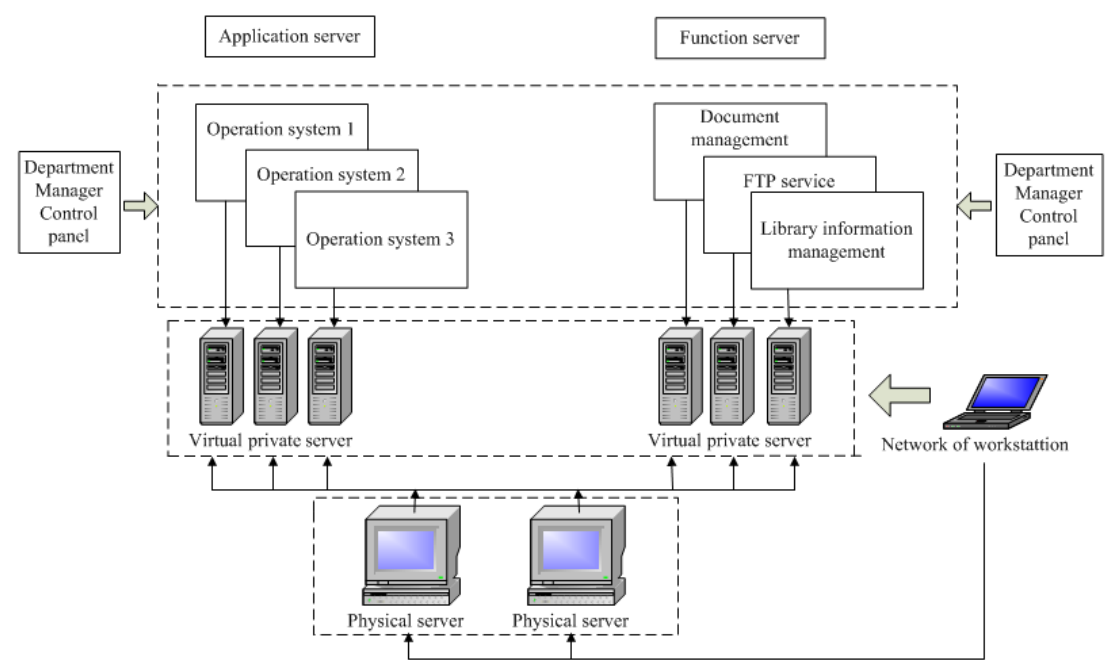

Figure 1. Cloud computing Campus Information Center Master Plan

\section{Function Modules}

According to the basic requirements for the data resources of the digital campus, the function architecture of cloud computing information resources center should be composed of the security management module, automatic service management module, virtualization management module, information resource management module, storage backup module, cloud portal management module and other modules.

\subsection{Security Management Module}

(1) Realize the authority management of tiered user. Establishing perfect user management based on LDAP standard, can carry on the management of weather users can use computing resources or not, computing resources that has been the use and related administrative authority and the relationship between the application projects. 
User management module should match with the server resources, storage resources and network resources, and should be consistent with the whole staff directory system.

(2) The cloud security level management. Realize the cloud application level security division and data security, application security, access control, and monitoring [6] of different levels, and meet the requirement of the difference of security for the applications in the cloud. There are relevant design server, storage, and network security monitoring in every level, then make sure that the end user's application runs reliable and credible.

\subsection{Automatically Service Management Module}

(1) Realize service template management. Service template is defined for the standardization and standardization of IT resources, including the server configuration, quantity, memory size, network configuration, operating system and application software configuration etc. Take some applications as an example, the resource requirements is standardized described as "a 2 virtual CPU, 2 GB memory, 15 GB storage for virtual servers, and install the Windows operating system, the DB2 database, WebSphere middleware software". The management module realizes customization such as adding, modifying, deleting, et for all service templates.

(2) Realize the automation management template. According to service template, you can choose the parameters of software and hardware resources needed by application to conduct automated deployment [7].Through service template management, according to the defined template, also can change the parameter, then, realize the automatic system deployment.

\subsection{Virtualization Management Module}

(1) Realize the pooling resources management. With virtualization technologies such as servers, storage, network and so on, according to different standards, organizing the cloud resources into different resource pool, can quickly and easily automatic customize resources needed by the upper applications in the resource pool. This module should widely support various servers, storage, and network. According to different standards, we can define different resource pools: according to the performance definition, resource pools can be divided into high performance computing resources pool, high storage capacity resource pool, minicomputers resource pool, etc. According to the function definition, resource pools can be divided into the database resource pool, middleware resource pool. The definition mode of the resource pool is flexible, convenient. Through the pooling management module, the administrator need not consider server, storage and network configuration [8].

(2) Realize related management scheduling of the virtual machine. Virtualization technology runs through the architecture of cloud computing model. It can be said that because of the virtualization technology, cloud computing become improved and mature. Virtual machine management module mainly realizes the centralized management of all kinds of virtual machine. According to the system resources, conduct the unified deployment, switch, load and migration, and realize zero downtime of application system by a variety of load balancing and cluster technology. 


\subsection{Information Resource Management Module}

(1) Realize the management of all information resources in the cloud. The module can add, delete, modify, configure and automatically recognize [9] the information resources in the cloud such as network, storage, servers, software, databases and so on by extensible equipment resource management interface. Information resource management module provides a Web access interface for the outside. The background is mainly composed of the information resource database, middleware module and information resources management interface.

(2) Realize the performance monitoring of information resources in the cloud. It can mainly realize monitoring the physical device, virtual host and application system, including monitoring all kinds of main operation parameters of the operating system, monitoring particular file system, monitoring specific processes, monitoring the virtual resources and monitoring the operating of the application system. Resource monitoring module should be able to monitor all physical devices in the resource pool and virtual host.

\subsection{Storage Backup Module}

(1) Data disaster recovery replication management. On the basis of the application service virtualization, we use synchronous replication technology and related products to automatically or manually realize application fault migration of related business, data protection, backup, restore, and other functions, and ensure data synchronization.

(2) Storage virtualization management. Realize centralized and unified management of heterogeneous storage devices, and provide service for virtualized storage.

\subsection{Cloud Portal Management Module}

Cloud services portal is that users use the interface of cloud computing resources, and provides a directory of all the available services on the existing cloud services portal, and products the unified scheduling and management of various functional modules on the background.

\section{Analysis of the System Safety}

Cloud computing information resource center products unified management of software and hardware resources in digital campus. It stores a large number of data of digital campus, involving many departments, systems and business, and should meet high security requirements for resource center.

\subsection{Hierarchical Security Strategy Construction}

First of all, data security center is hierarchical. Therefore, we also need to adopt a hierarchical security policy on safety, including the Internet access layer, convergence layer, business layer and operational management. According to different security problem, there are different security strategies in different levels. For Internet access layer, the main threat is a DDoS attack. The main problem of DDoS attack is to run out of bandwidth [10]. The main measures to solve this problem is a more mature technology, it is flow cleaning. It will perform the deployment of the flow cleaning in the data center, and export. For the safety of business access layer, it mainly directed 
against the host resources. On one hand, perform the virus protection, establish a concentrated virus library, virus engine, periodically perform antivirus and cleaning to host; on the other hand, perform the periodic safety assessment. According to the result of safety evaluation, constantly update protection means. Finally, perform reinforce security to the host itself.

For convergence layer, one is to perform access control, product the effective isolation protection to the unreliable access; The second is to perform effectively supplement to the preventing intrusion system; The three is to perform security reinforcement to the network equipment, that is, equipment of the bearing level, including strictly control access, and the limit to the open service of network equipment itself.

User terminal management includes an assessment of terminal security. The results of the safety assessment determine whether to allow others to access. In addition, we need periodically audit the users' behavior and users' terminal. According to the statistical analysis of a long-term, make a safety analysis of the users' use habit, so as to improve the ability of security protection.

The safety of the operations management is divided into two levels: one level is the security protection of the remote access form. Another level is security protection for the local operations staff. Remote access protection of operations management controls the access permissions to the user, access ability, the bandwidth by setting the security control gateway. The remote way of VPN, includes SSL VPN. There are different forms according to different requirements.

Finally we concern new technology security. In view of the cloud computing, it mainly includes virtualization security protection, safety protection for storage and network security protection. Aiming at the CDN, it mainly protects the server from DDoS attacks, to improve the reliability of the server.

\subsection{Account Access Security Construction}

Cloud computing management center can use the separate network segments. The external cannot access the equipment in the cloud-computing center through the network. Each physical server management port is connected to the management VLAN or physical independent network through a separate physical network card, which can effectively avoid external unauthorized users damage the underlying architecture of the cloud-computing platform through business network. In addition, in the business access layer, it adopts the traditional access control measures. And identify the legitimacy of external users' access through identification authentication, certificate authentication, network access and other access. And award it its rightful role permissions. Then access the information resources in the cloud-computing platform. In the cloud computing management center, can award different control permissions to different management account in management platform. It can restrict users only for a resource group, a virtual machine or a network port group to control authority, and perform logging to the operation of the visitors. Unless it is the super administrator, general administrator is unable to delete records. History $\log$ records can be exported and archiving. On the management platform, it can also be integrated with $\mathrm{AD}$, using domain account, and gives different permissions to the account. In the resources allocation platform, according to different users' identity, different business characteristics, we can also make different access control strategy, and distribute different performance of resource pool: distribute resources with high availability to important business, and distribute resources with poor availability to the secondary business. 


\subsection{Disaster Redundancy Safety Construction}

For each business users of the school, availability is first. Hardware failure, software failure all will affect the system availability. Cloud computing should be able to provide high quality, high efficiency, the availability of services which satisfies the customer. Therefore, the system has the ability of fault tolerance and disaster.

(1) Networking: Adopt double link access in the local area network, and avoid single point of failure. In a network device or link failure cases, virtual gateway can drift to another equipment of network in parallel by the network high availability technology.

(2) Server: In view of the server hosting virtual computing resources, we can configure cluster system in the cloud resource center, and product high availability cluster for two weavers. Using virtualization software HA function, in a single virtual machine or physical machine malfunction, the virtual machine can seamlessly migrate to other physical machines in the cluster.

(3) Storage: The raid technology ensures data security of the hard disk level; Adopt double controller and store their own hardware module redundancy technology, which can guarantee the business system without interruption in a single module failure. Multimodal technology is used to connect to the master, which ensures the safety of the link.

(4) Disaster mechanism: In the single computer room environment, with the restriction of electric power facilities, refrigeration facilities and other force majeure factors (such as fire, earthquake, etc.), the paralysis of data center may happen. The construction of disaster preparedness center can effectively prevent the occurrence of the general disaster situation [11]. In master data center failure cases, we can start the spare computing resources in emergency center, to ensure continuity of the service and high availability of the data.

(5) Software level: After migrate the business system to the cloud computing center, it can easily execute snapshot, backup on operating system, data storage. And online backup will not affect the normal running of the business. Develop detailed backup strategy, and regularly perform the machine backup to the virtual machine files and key business system. In the event of a disorder in the system, the backup recovery mechanism can quickly restore to a certain time point.

\section{Information Resource Integration Demonstration}

The function of cloud computing information resource center is the distribution of computing tasks in resource pool constituted by a large number of networks, servers, storage and so on. It can make all kinds of application system access computing power, storage space and information according to the need. At the same time, resource pool can dynamically scale to meet the requirements of the application system operation. Therefore, resources need to be integrated. Through the establishment of resource integration model, improve the model speed so as to realize resource integration. At the same time, virtualization resources pool computing resources service for the business system.

\subsection{Resource Integration Model is Established}

The model structure of the resource integration model realized in this paper is shown in Figure 2 below: 


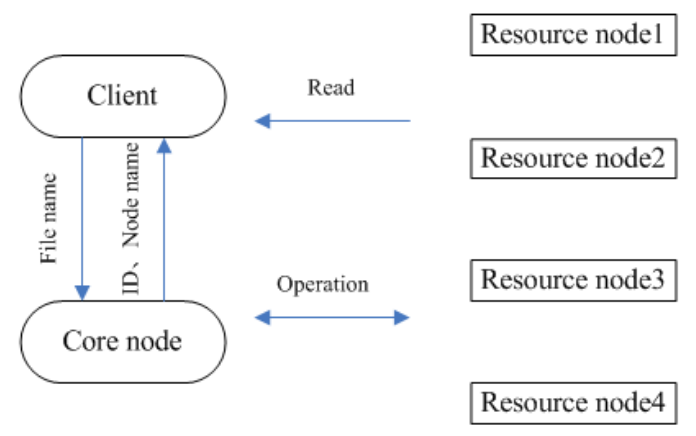

Figure 2. Resources Integration Model Diagram

Through the above figure we can clearly see the whole process of teaching data under the integration of cloud computing: the client sends a request to core node through the file name, then the core node finds the resources in the resource nodes through the file name. The client directly read after finding the resources.

This model can be divided into six levels, namely the data layer, cloud client, management layer, application layer, presentation layer and hardware layer. Cloud client refers to the customer's access to the software of this system. In general, the client is a Web server, such as Internet explorer, Firefox, Chrome, etc.

The application layer is composed of the Portal Server and Portlet container. Portal Server receives the cloud client's HTTP request, then calls the Portlet in the Portlet container, and returns the content data generated by Portlet back to the client. A portal page is normally composed of several Portlets. Every Portlet shows the corresponding service with cloud computing, cloud services.

Management layer is used to realize various cloud services, such as user maintenance, integration of data, management of resources and their directories, etc.

Data layer is used to make the data inside the hardware layer virtual, and be virtualized into virtual file, and then perform file-classified storage, such as the distributed file system of Hadoop.

There are lots of storage resources in the hardware layer, including server, etc.

\subsection{Improve Model Speed}

Campus information resources often have hundreds of $\mathrm{G} \mathrm{M}$ or even $\mathrm{T} \mathrm{M}$. In order to guarantee the running speed of the model, this paper joined the multi-objective service operation method. It can choose appropriate nodes according to the priority to satisfy the speed optimization [12]. Mainly consider the following several parameters:

(1) Completion time: the total time cloud computing requires. This article estimates based on the request times, previous running time and the estimated running time. Then the request $\mathrm{Si}$ estimated the finish time TC:

$$
\begin{gathered}
\mathrm{Tc}=\mathrm{Qs}=\mathrm{Tc}+\sum_{k=1}^{Q L d} T k c \\
\mathrm{Tc}=\frac{\operatorname{Tin} i+\sum_{m=1}^{N m} T n}{N m+1},(N m \neq 0)
\end{gathered}
$$


Among them, $\mathrm{QL}_{\mathrm{d}}$ represents time needed in node $\mathrm{d} . \mathrm{T}_{\mathrm{C}}$ represents the expected computing time of $S_{i}$. The expected computing time of $S i$ is $T_{\text {ini }}$, the value is the average value of the computing time before this request. $\mathrm{Nm}$ represents the request times, and $\mathrm{T}_{\mathrm{n}}$ represents the nth computing time of $\mathrm{Si}$. The more times $\mathrm{Si}$ finishes, the closer the value of $\mathrm{T}_{C}$ to real service completion time. The smaller the value of Tc, the faster the service can finish. We find the node with smallest value of Tc, and can run in the node, which can ensure real-time services.

(2) Load balancing: if the load of some node in the cloud is more than the maximum load limit it can bear, the overall performance of the platform will reduce. Therefore, we need schedule the service to nodes whose load is not heavy to run as far as possible, to make the load balanced. In the experiment, set a denial service critical value for each node. When the load of device node exceeds the critical value, deny the service. The critical value computation formula is as follows:

$$
\text { TLV }=\{\text { TLVcup , TLVmem, TLVstor, TLVnet }\}
$$

(3)Load Baseline Algorithm Based on Radial Basis Function(RBF) neural network. Baseline calculation is important in the field of network monitoring, especially in C3IC. this article gives a baseline algorithm based on Radial Basis Function to calculate the baselines, combined with ant swarm intelligence algorithm to achieve the parameters(the hidden units, prior hyper-parameter and noise hyper-parameter,). the pseudo-code is shown as following:

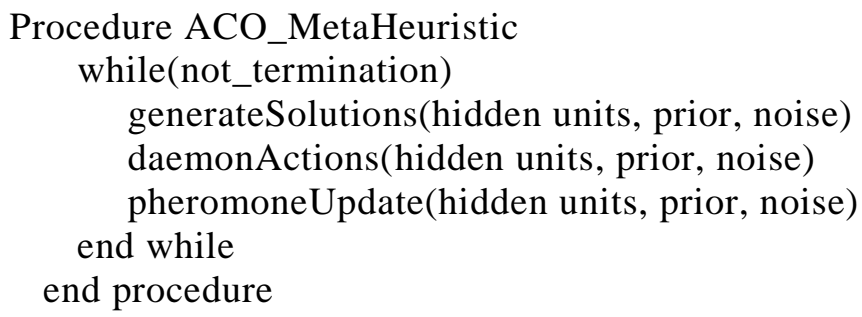

\section{Experiment}

To test the proposed Baseline Algorithm Based on RBF, we collect a sever in our center for around 455 days' load records. The first 400 days' records are taken as the training set, and the rest 55 days as the test set, shown in Figure 3. In which, 20\% error bar is set as the range of tolerant level. Our RBF algorithm is compared with Multi Laxer Perception(MLP) and Back Propagation(BP) neural network algorithm. In these neural network algorithms, the hidden units are set as 3, the prior hyper-parameter is set as 0.01 , and the noise hyperparameter is set as 50. And the training cycles in inner loop is set as 500. Then we get the following result shown as Figure 4. The y-axis means the load value, and the x-axis is the sequence of time. It is clear than our RBF algorithm is better than the MLP and BP algorithms, with more close to the background value. 


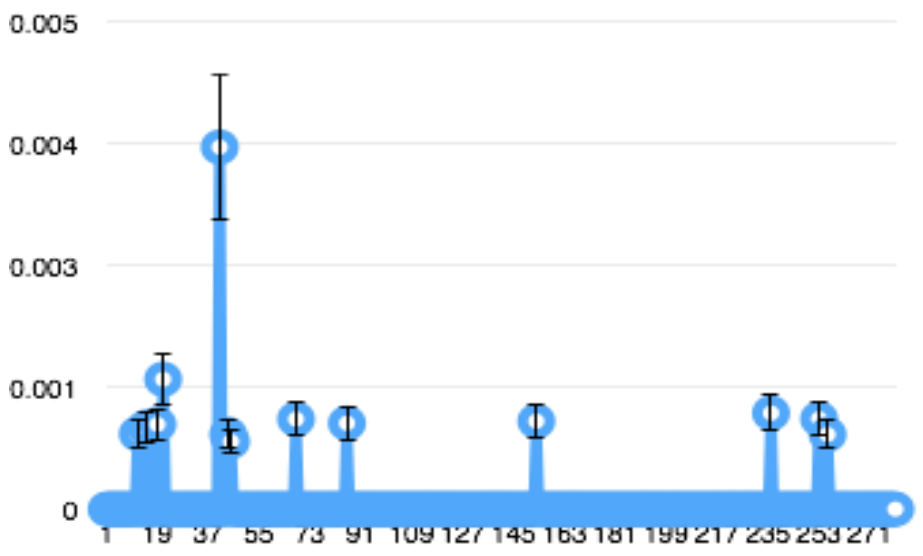

Figure 3. Resources Integration Model Diagram
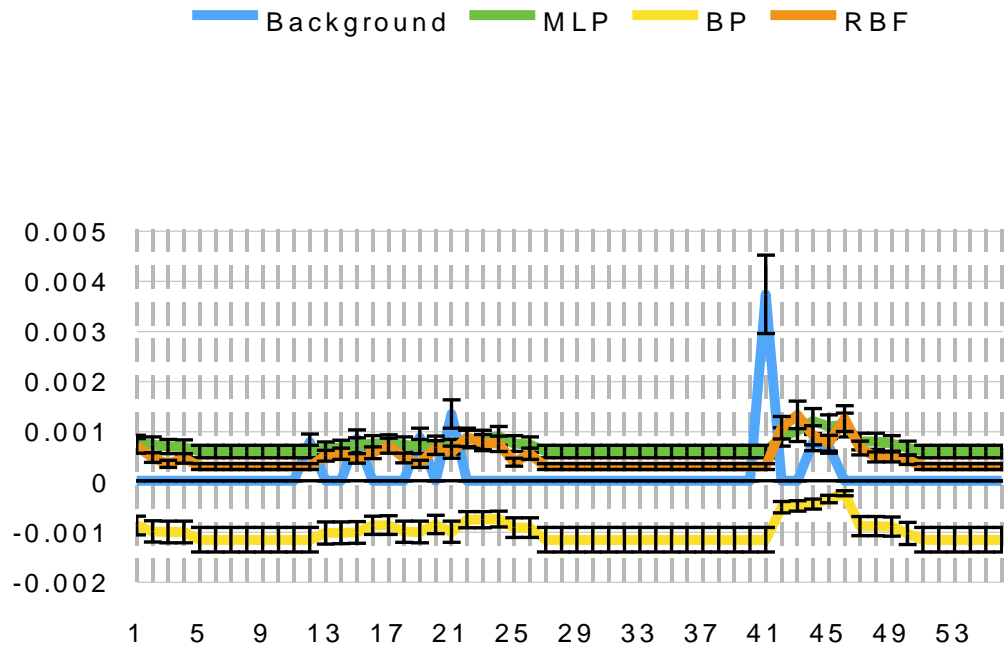

Figure 4. Baseline Algorithm Comparison

\section{Conclusion}

C3IC is the new trend of digital-campus data resources sharing platform. In C3IC, the hardware and software resource, such as hosts, storage, nets and applications, can be integrated as a whole. And through the private clouds, some basic serves, such as data storage, disaster technical backup, and runtime environment, can also be provided. With the effective monitor and management, the units in digital campus can be provided with computing-resources rent service in virtualization. Thus it is practice for the on-demand rationing of the resources, low unit of resource, and digital-campus innovation.

\section{Acknowledgment}

The authors would like to thank the computer science major teaching supervision committee project fund of technical and vocational college under Ministry of Education 
(JZW590112KC35), the National Natural Science Foundation of China (No. 61272315, No.60842009), Zhejiang Provincial Natural Science Foundation (No.Y1110342) and Zhejiang provincial science and Technology Department of international cooperation project (No.2012C24030).

\section{References}

[1] W. Bo, C. Yi and Y. Weiping, "Production-study-research cooperation study based on cloud computing", Science \& Technology Progress and Policy, vol. 30, (2013), pp. 1-4.

[2] Z. Yulong, "Optimization and Research of University Teaching Resource Integration Model Based on Cloud Computing", Bulletin of Science and Technology, vol. 29, (2013), pp. 201-204.

[3] G. Y.ia and G. Jinggang, "Universities virtuallaboratory teaching research based on cloud computing", manufacturing automation, vol. 3, (2013), pp. 87-90.

[4] T. ShuiLong, "In the research of the digital archive construction based on cloud computing", China Archives, (2013), pp. 60-63.

[5] C. Chen, "Digital library virtualization platform based on cloud computing credible management strategy research", LIBrary Theory and Practice, (2013), pp. 82-85.

[6] S. Feiju, "Mobile library construction analysis based on cloud computing", E-Business, (2013), pp. 54-55.

[7] J. Guodong, "Research on construction of digital campus cloud computing resource center", Experimental Technology and Management, vol. 9, (2013), pp. 212-216.

[8] Z. Haiyan, Z. Wende and W. XiNing, "Based on SOA and cloud computing fusion of information system integration research colleges and universities", The ChineseJournal of ICT in Education, (2013), pp. 47-50.

[9] W. Manying and G. Jianming, "Multi-evaluationSystem of Graduation Practice Under Cloud Computing Environment", Bulletin of Science and Technology, vol. 8, (2013), pp. 225-228.

[10] R. Min, "Application and improvement of key agreement protocol in could computation environment", Journal of Computer Applications, vol. 33, (2013), pp. 2835-2837, 2864.

[11] C. Huifen and L. Qingwu, "Application of Cloud Computing in the management of Computer Labs in Colleges", Research And Exploration In LaboratoRY, vol. 32, (2013), pp. 213-216.

[12] C. Chunling, P. Yu, and Z. Dengyi,"Energy saving resource scheduling algorithm in cloud environm en", System s Engineering and Electronics, vol. 11, ed, (2013), pp. 2416-2423.

\section{Authors}

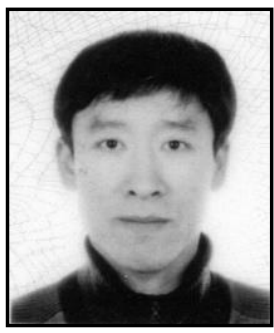

Zhou Zili, Corresponding author. He received the M.S. from Zhejiang University of Technology. Now he is the senior engineer, associate professor of Zhejiang Institute of Mechanical \& Engineering, $\mathrm{He}$ is principally engaged in computer information processing and application of technology.

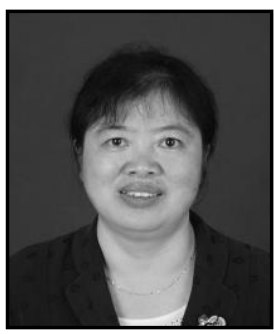

Lu Huijuan, she received her Ph.D. and B.S. from China University of Mining \& Technology, the M.S. from Zhejiang University. Now she is the Professor of China Jiliang University. She is the executive director of CCF and the member of China cloud computing Expert Committee. She is principally engaged in cloud computing, pattern recognition, bioinformatics, data mining. 